\title{
Anabases
}

ANABASES Traditions et réceptions de l'Antiquité

\section{Approche historique et spatiale de la Phénicie hellénistique : cités, territoires, modélisation}

\section{Élodie Guillon}

\section{(2) OpenEdition}

\section{Journals}

Édition électronique

URL : http://journals.openedition.org/anabases/5509

DOI : 10.4000/anabases.5509

ISSN : 2256-9421

\section{Éditeur}

E.R.A.S.M.E.

\section{Édition imprimée}

Date de publication : 20 octobre 2015

Pagination : 258-264

ISSN : 1774-4296

\section{Référence électronique}

Élodie Guillon, «Approche historique et spatiale de la Phénicie hellénistique : cités, territoires, modélisation », Anabases [En ligne], 22 | 2015, mis en ligne le 20 octobre 2018, consulté le 21 octobre 2019. URL : http://journals.openedition.org/anabases/5509 ; DOI : 10.4000/anabases.5509 


\title{
L'Atelier des doctorants
}

\section{Approche historique et spatiale de la Phénicie hellénistique: cités, territoires, modélisation}

\author{
Élodie GuILlon
}

À l'été 332 av. J.-C., Alexandre le Grand parvient à occuper la cité de Tyr après sept mois de siège, marquant ainsi la fin de la conquête de la Phénicie ${ }^{87}$. Au contraire de Tyr, les autres cités phéniciennes se sont rendues au conquérant. Le fait que ces cités se livrent sans combattre et qu'elles transforment dans la foulée leurs institutions politiques, abandonnant la royauté au profit d'un régime civique ${ }^{88}$, a longtemps été interprété comme une preuve du philhellénisme des Phéniciens et comme la marque de l'hellénisation de leur société.

Pourtant, depuis plusieurs années, la période hellénistique fait l'objet d'une profonde réévaluation scientifique qui a conduit à réviser le concept même d'hellénisation, hérité d'une lecture de l'époque adoptée dès le xix ${ }^{\mathrm{e}}$ siècle, qui fondait l'ensemble de ses interprétations sur une vision unilatérale de la dynamique entre Grecs et Proche-Orientaux. En effet, suite aux travaux de J.G.Droysen (I808-1884) ${ }^{89}$, l'attention des historiens s'est centrée sur les rapports culturels entretenus par les Gréco-Macédoniens et les habitants du Proche-Orient, pensés au miroir du colonialisme contemporain: les parallèles furent alors établis entre les colons européens et les troupes d'Alexandre d'une part, entre les Africains ou les Asiatiques, et les barbares de l'Antiquité d'autre part ${ }^{90}$; l'époque hellénistique devint ainsi un tournant culturel, négocié par les Grecs apportant la civilisation

87 Arrien, Anabase d'Alexandre, II, I5-24; Diodore, Bibliothèque historique, XVII, 40-46; Polyen, Stratagèmes, IV; Quinte-Curce, Histoire d'Alexandre le Grand, IV, 2-3.

88 M. Sartre, D’Alexandre à Zénobie. Histoire du Levant antique, IV siècle av. J.-C.-III siècle ap. J.-C., Paris, 20oı, p. I5o. Le changement de régime est visible sur les monnaies, sur lesquelles une "ère du peuple» commence dans la première moitié du $\mathrm{III}^{\mathrm{e}}$ siècle dans toutes les cités.

89 J. G. Droysen, L'Histoire de l'Hellénisme (nouvelle édition préfacée par P. Payen), Grenoble, 2005.

90 P. Briant, Rois, tributs et paysans. Études sur les formations tributaires du Moyen-Orient ancien, Paris, I982, p. I83. 
à l'Orient. Les fondations de cités et la diffusion du grec et des savoirs s'exprimant dans cette langue furent regroupés sous le label d'“hellénisme» (Ellenismus).

La dichotomie Grecs/Orientaux perdura jusque dans la seconde moitié du $\mathrm{xx}^{\mathrm{e}}$ siècle, car la décolonisation, si elle invita à revisiter les dynamiques culturelles de cette période, ne remit pas en question ce "grand partage»; il favorisa une interprétation en termes de contre-acculturation et de résistance culturelle ${ }^{91}$. Ce n'est que dans les années I980-I990, en particulier avec le processus de réflexion autour du multiculturalisme aux États-Unis et la chute de l'urss, que s'est développée la vision d'un monde multipolaire aux logiques culturelles complexes et pluridirectionnelles. L’hétérogénéité des contextes hellénistiques déboucha sur une remise en question de la notion excessivement univoque d'hellénisation, peu à peu délaissée au profit de concepts plus riches et plus adaptables, tels que celui de “transfert culturel ${ }^{92}$ ».

Le dossier des cités phéniciennes n'a émergé que récemment, avec le réexamen des corpus de sources ainsi qu'une première tentative de synthèse sur ses paysages religieux d'époque hellénistique ${ }^{93}$. Dans le cadre de ce processus, les historiens du monde phénicien ont eu tendance à considérer le territoire des cités davantage pour en établir les limites que pour comprendre son organisation. Ils ont eu du mal à se départir des cadres territoriaux proprement grecs, reposant sur les notions d'asty et de chôra; ils se sont insuffisamment affranchis des typologies modernes (ville/campagne, bourg, village, etc.) qui traduisent une conception contemporaine de l'espace.

Or ce dernier peut être défini comme un champ d'expression des sociétés, au même titre que les discours et les écrits qu'elles produisent ${ }^{94}$. L'objectif de notre thèse ${ }^{95}$ a donc été de repérer et d'analyser les spécificités phéniciennes en matière de pratiques spatiales,

91 M. WaelKens et al., “Indigenous versus Greek Identity in Hellenistic Pisidia: Myth or Reality? ”, in M. Dalla Rrva (éd.), Meetings between Cultures in the AncientMediterranean. Proceedings of the I7th International Congress of Classical Archaeology, Rome 22-26 sept. 2008, Bolletino di Archeologia on-line, 2010, p. 19-23.

92 J.-C. Couvehnes, B. Legras (dir.), Transferts culturels et politique dans le monde hellénistique. Actes de la table ronde sur les identités collectives (Sorbonne, 7février 2004), Paris, 2006, p.5-8. Élaboré vers I985 par M.Espagne et M.Werner, pour parler des relations franco-allemandes, le concept a été rapidement repris par les antiquisants, pour sa grande plasticité qui permet d'englober un ensemble de notions polémiques, dont celle d'hellénisation, afin de les dépasser.

93 Pour Sidon, C. Apicella, Sidon aux époques hellénistique et romaine, Tours, 2002. Pour Arwad, F.Duyrat, Arados hellénistique. Étude historique et monétaire, Beyrouth, 20o5. Pour la Phénicie, C. Bonnet, J. Aliquot (dir.), La Phénicie hellénistique. Nouvelles configurations politiques, territoriales, économiques et culturelles. Actes du colloque international, Toulouse, I8-2o février 20I3, Lyon (sous presse).

94 F. Toulze, “Centre et périphérie à Rome », Uranie 3, 2003, p. 87-II8.

95 La thèse de doctorat intitulée Les arrière-pays des cités phéniciennes à l'époque hellénistique ( $\boldsymbol{V}^{e}$ siècle-ter siècle av. J.-C.). Approches historiques et environnementales d'une aire géoculturelle et dirigée par Corinne Bonnet et Jean-Michel Carozza a été soutenue le 8 novembre 2013 à l'UT2J. 
Figure ı: Carte des itinéraires pédestres modélisés en Phénicie ( Élodie Guillon)

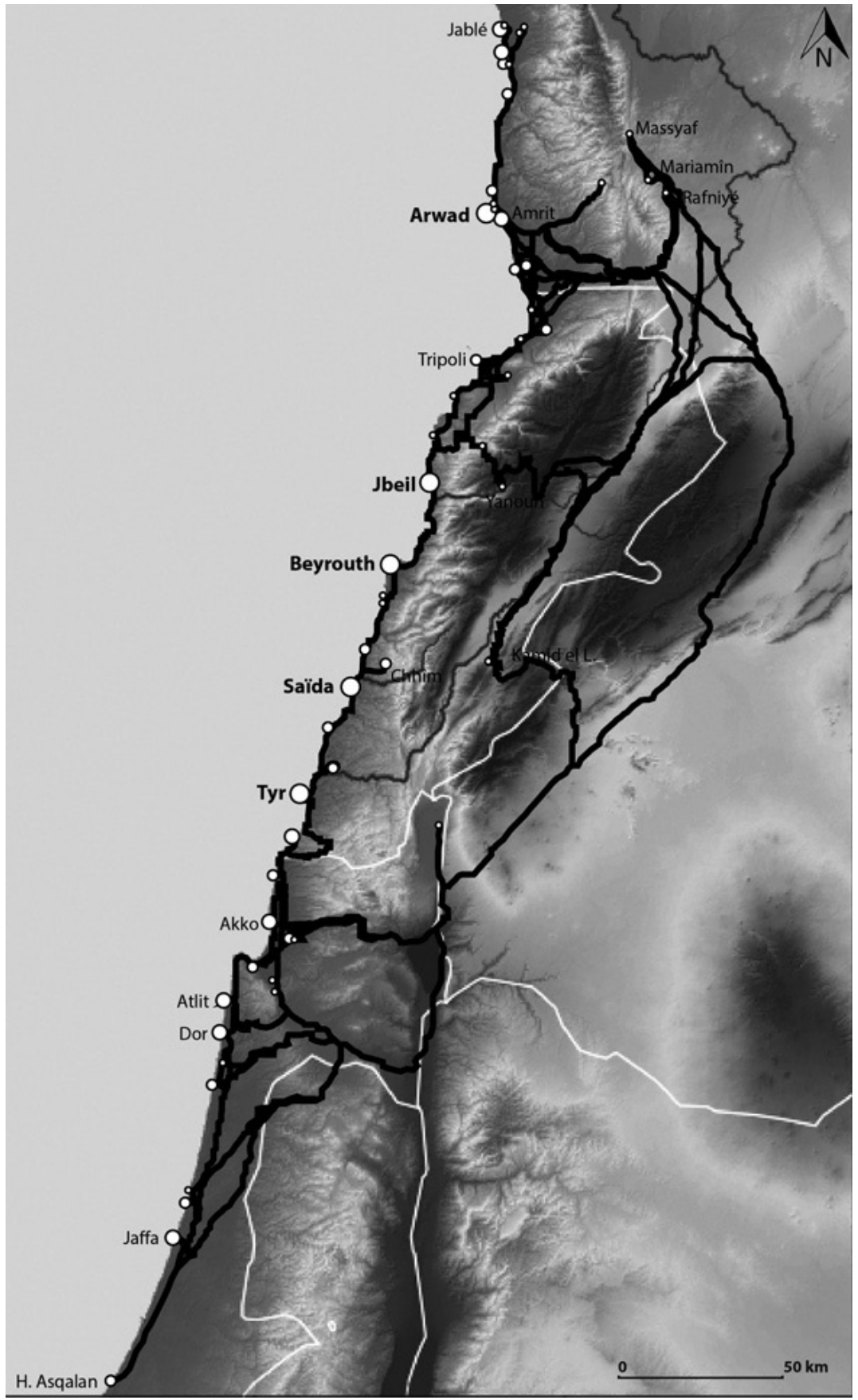


c'est-à-dire leur façon d'organiser les cités, d'administrer les territoires et d'y circuler. En l'absence de littérature phénicienne, cette manière spatiale de discourir constitue une fenêtre ouverte sur la société et les conceptions phéniciennes des rapports de l'homme à son environnement.

\section{L'espace phénicien antique: prise en compte et développement de l'objet d'étude spatial}

Si les chercheurs n'ont jamais nié le fait que les cités phéniciennes possédaient un territoire, ils ont toutefois tardé à le considérer autrement que comme une bande côtière limitée par la montagne, occupée par des établissements urbains entièrement tournés vers la mer ${ }^{96}$. En I995, cependant, la contribution de J.-F.Salles ${ }^{97}$, dans l'ouvrage de synthèse intitulé La civilisation phénicienne et punique. Manuel de recherche, marqua une rupture dans la définition traditionnelle de la Phénicie. L'auteur proposa en effet de considérer l'espace phénicien non plus seulement comme un arrière-plan, un port de départ vers la Méditerranée, mais comme un sujet de recherche à part entière au sein des études phéniciennes. C'est dans cette logique que les cités d'Arwad et de Sidon, en particulier, ont fait l'objet de synthèses historiques à partir de l'ensemble des sources disponibles. Les cités y sont étudiées comme des entités cohérentes, dont les territoires sont occupés par différentes agglomérations interconnectées et structurés par des voies de circulation. Dans la même perspective d'un renouvellement des échelles de réflexion sur l'espace phénicien, des programmes de prospections archéologiques se sont mis en place en Syrie et au Liban pour réfléchir au fonctionnement de microrégions, telles que le Akkar ou la Béqaa ${ }^{98}$.

Le corpus phénicien, il faut le concéder, est maigre, comparativement à ceux de la Grèce et des aires puniques, pour lesquelles les études spatiales se sont développées depuis plus de trente $a n s^{99}$. L'étude des territoires des cités phéniciennes a donc dû s'appuyer sur une méthodologie différente de celles utilisées dans les études grecques et puniques, qui met à contribution des outils d'analyse tirés de la géographie capables de s'adapter aux contraintes d'une documentation disparate et essentiellement archéologique. L'espace

96 Cette représentation provient d'une description de l'implantation d'établissements phéniciens en Sicile dans Thucydide, Histoire de la guerre du Péloponnèse, VI, 2.6.

97 J.-F. Salles, «Phénicie», in V. Krings (éd.), La civilisation phénicienne et punique. Manuel de Recherche, New York, I995, p.553-582.

98 Tous les résultats ne sont pas publiés, mais, parmi les plus récents, pour le Akkar, K.BARTL, "Akkar Survey 1997. Archaeological Surface Investigations in the Plan of Akkar/Northern Lebanon. Preliminary Results », BAAL 3, I998-ı999, p. I69-I79. Pour la Béqaa: M.Heinz et al., "Kamid el-Loz in the Beqa'a plain/Lebanon. Continuity and Change in the Settlement of the Region ", BAAL 5, 200I, p.5-9I.

99 Récemment, sur l'occupation du territoire: S. FACHARD, La défense du territoire. Étude de la chôra érétrienne et de ses fortifications, Athènes, 20I2; sur l'agriculture grecque: L. Foxhall, Olive Cultivation in Ancient Greece. Seeking the Ancient Economy, Oxford, 2007. Pour le monde punique, P. Van Dommelen, C. Gómez Bellard (dir.), Rural Landscapes of the Punic World, Londres, 2008. 
devient alors le point de départ d'une enquête qui, en mobilisant l'ensemble des sources disponibles, vise à le comprendre et à en tirer des informations de type historique.

\section{Les outils géographiques au service des études historiques}

Cette enquête s'est inspirée d'un travail collectif mené en Lodévois (Hérault), reposant sur un corpus documentaire hétéroclite de sites archéologiques datés des premiers siècles de notre ère jusqu'à l'époque moderne ${ }^{100}$. Ces sites ont été classés hiérarchiquement suivant les époques ${ }^{101}$ pour modéliser des réseaux de fonctionnement constitués de pôles et d'établissements secondaires reliés aux premiers. Si le sens des interactions est déterminé par le modèle lui-même, leur caractérisation - les types et l'intensité des échanges - revient aux archéologues et aux historiens qui analysent les résultats selon leur connaissance des différents contextes ${ }^{102}$.

Le corpus phénicien traité, composé de 5r sites archéologiques - avec leurs structures architecturales, l'ensemble du mobilier découvert, et les mentions dont ils font l'objet dans les sources grecques et romaines -, présente le même caractère lacunaire et hétéroclite que le corpus lodévois. Partant, les processus de traitement de la documentation ont été adaptés à la Phénicie, d'autant plus que le postulat de départ est le même qu'en Lodévois: les sites ont été liés par des relations impliquant des complémentarités et des dépendances à une époque historique. L’ensemble du corpus phénicien a donc été enregistré dans une base de données qui a permis d'organiser les données et de classer les 5i sites hiérarchiquement avant qu'ils soient intégrés à un modèle gravitaire ${ }^{103}$. La modélisation spatiale a abouti à créer des réseaux supposés d'interactions entre les sites, ainsi que des itinéraires de déplacement pédestre tenant compte de la topographie de la zone (fig. I).

100 L. Nuninger et al., “La modélisation des réseaux d'habitation en archéologie: trois expériences »,M@ppemonde 83, 2006,p.5, I0-II, I6 et I8.

101 Le classement s'appuie sur la surface d'occupation estimée des sites et sur des analyses statistiques multivariées. L. Nuninger et al., «La modélisation », p. 6, Io et I9.

102 L. Nuninger et al., “La modélisation », p.23.

103 Le modèle gravitaire, tiré de la théorie de la gravitation universelle énoncée par Newton en I687, est utilisé pour formaliser et étudier les interactions entre deux entités spatiales (deux villes par exemple). P. Garmy, Villes, réseaux et systèmes de villes. Contribution de l'archéologie, Paris, 20I2, p. I9o-I93; É. Guillon, “L’organisation spatiale des arrièrepays de la Phénicie hellénistique ( $\mathrm{IV}^{\mathrm{e}}-\mathrm{I}^{\mathrm{er}} \mathrm{s}$. av. J.-C.). Apport de la modélisation spatiale de données archéologiques », Cybergéo (à paraître). 


\section{Résultats de la modélisation et nouvelles hypothèses sur la Phénicie hellénistique}

Si les résultats sont un strict reflet de l'état du corpus, ils participent néanmoins à une relecture de la période hellénistique. Les bouleversements qu'elle engendre sur divers plans (politique, économique, religieux, etc.) ne semblent guère affecter les réseaux modélisés qui paraissent pérennes, ne subissant de changements qu'à l'échelle locale. Cette stabilité s'explique si l'on considère les reliefs qui marquent fortement la région et la grande résilience de ses pôles (les cités). Les réformes politiques et économiques menées au Proche-Orient par les souverains hellénistiques ne sont cependant pas restées sans conséquence, comme en témoignent les sources archéologiques et numismatiques. S’il est encore impossible d'identifier de façon sûre et de quantifier les flux marchands et humains empruntant les réseaux, on est amené à s'interroger sur ces derniers, qui se modifient probablement davantage (en nature et en intensité) que les voies empruntées à l'époque hellénistique ${ }^{104}$.

Par ailleurs, une nouvelle vision de la Phénicie a émergé. Les réseaux montrent en effet l'importance des connexions entre la côte et l'arrière-pays. La modélisation des itinéraires pédestres fait en outre ressortir l'intégration de la vallée de l'Oronte et de l'AntiLiban aux circulations phéniciennes plus étendues qu'on ne le supposait. Ces itinéraires mettent également en avant le rôle des vallées et des cols qui concourent à former un ensemble cohérent permettant la communication entre les sites côtiers et les sites montagneux, mais également entre les sites d'arrière-pays sans passer par le littoral. Ces observations combinées avec l'analyse du mobilier archéologique aboutissent à qualifier la Phénicie et ses cités comme des territoires méditerranéens, au sens défini par P.Horden et N.Purcell ${ }^{105}$. En effet, dans leur fonctionnement spatial, les cités phéniciennes ressemblent à d'autres cités méditerranéennes, comme celle d'Érétrie, récemment étudiée par S. Fachard ${ }^{106}$ : elles sont un espace structuré et cohérent constitué de petits ensembles interconnectés - les microrégions caractérisées par un paysage, une cohérence politique ou économique.

Finalement, en s'attachant à exploiter et à valoriser la documentation issue des sites phéniciens par une approche scientifique inédite, cette recherche a révélé un espace phénicien à double dimension, à la fois terrestre proche-orientale, et maritime méditerranéenne. Le grand potentiel de la démarche adoptée, découlant de la possibilité d'actualiser et d'adapter le modèle, permettra de travailler, avec les mêmes objectifs, sur d'autres zones de peuplement et de mobilité phéniciennes, à commencer par le territoire

104 S. Elaigne «La vaisselle à vocation régional de Phénicie hellénistique: aperçu à partir des assemblages de Beyrouth », in C. Bonnet, J. Aliquot (dir.), La Phénicie. La publication progressive des études des céramiques hellénistiques devraient permettre de caractériser davantage les échanges et les flux.

105 P. Horden, N. Purcell, The Corrupting Sea: a study of Mediterranean history, Oxford, 2000, p.8o. Les microecologies désignent des terroirs et des paysages à petite échelle, variant selon les régions.

106 S. FACHARD, La défense du territoire. 
insulaire d'Ibiza ${ }^{107}$, afin de dépasser l'image d'un paysage phénicien urbain et maritime au profit d'une étude approfondie des occupations dans leur environnement.

Élodie Guillon

Université Toulouse-Jean Jaurès (UT2J) PLH-ERASMe - Maison de la Recherche

5, allées Antonio Machado

3ı 58 Toulouse Cedex 9

eguillon3ı@gmail.com

\section{Tradition de la pensée politique}

\section{Tragedy and History. About a Recent Book on Arnold Toynbee ${ }^{108}$ \\ Vito Limone}

The main aim of this book by Federico Leonardi is to propose a new view of Arnold Toynbee's philosophy of history, tracing its roots back to a little known lecture the British historian delivered in Oxford on May I920, The Tragedyof Greece. In fact, the book's appendix contains the first Italian translation of the lecture (The Tragedy of Greece. A Lecture Delivered for the Professor of Greek to Candidates for Honours in Literae Humaniores at Oxford in May I920, Clarendon Press, Oxford I92I). In this conference Toynbee sketched his view of the civilization, that later he applied in his masterpiece, A Study of History, whose twelve volumes were composed and immediately edited by Oxford University Press between I934 and ig6r. Critics always agreed that Toynbee was irenistic and they dismissed his mix of history and theology, whereas Leonardi puts forward that Toynbee was tragic and consequently to understand history is to recognise its tragedy and to seek an alternative, a reconcilation.

107 C'est l'objet d'un programme de recherche de deux ans financé par l'idex ATs Patrimoine de Toulouse: “Phéniciens et Puniques à Ibiza. VII ${ }^{\mathrm{e}}-\mathrm{III}^{\mathrm{e}}$ siècles av. J.-C. Patrimoine archéologique et modélisation spatiale».

108 Federico LeOnardi, Tragedia e storia. Arnold Toynbee: la storia universale nella maschera della classicità, Aracne, Atene e Gerusalemme 9, Roma 2014, I6o p. 